Sharif University of Technology
Scientia Iranica
Transactions E: Industrial Engineering
IRAN I I CA

Research Note

\title{
On enhanced sensitivity of nonparametric EWMA control charts for process monitoring
}

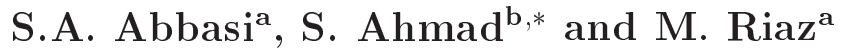 \\ a. Department of Mathematics and Statistics, King Fahd University of Petroleum and Minerals, Dhahran, 31261, Saudi Arabia. \\ b. Department of Mathematics, COMSATS Institute of Information Technology, Wah Cantt 47040, Pakistan.
}

Received 27 October 2014; received in revised form 10 November 2015; accepted 16 January 2016

\author{
KEYWORDS \\ Average run length; \\ Control charts; \\ Exponentially \\ weighted moving \\ average; \\ Extra quadratic loss; \\ Fast initial response; \\ Nonparametric; \\ Relative average run \\ length; \\ Time-varying.
}

\begin{abstract}
Control charts are popular tounderols for process monitoring and are mainly classified into memory and memory-less structures. Exponentially Weighted Moving Average control charts (EWMA) are widely used for the detection of small shifts in process parameters. The traditional design structures of these types of charts rely on the stringent assumption of normality. In practice, quality characteristics generally do not follow normality; hence, alternative charting structures are needed. Nonparametric control chart is one such alternative that do not depend on the mentioned distributional assumption(s). This study deals with nonparametric EWMA charts for an efficient detection of smaller shifts in location. We have investigated asymptotic, time-varying, and Fast Initial Response (FIR) based time varying control limits for nonparametric EWMA charts. We have considered two variants of FIR with time varying limits for nonparametric EWMA structure. A variety of run length properties have been investigated for performance evaluations. It is observed that FIR-based nonparametric EWMA charts with time-varying limits are quite sensitive to detecting smaller shifts, and they offer attractive run length properties. A real-life example is also provided to illustrate the application of the time-varying and FIR-based nonparametric EWMA control charts.
\end{abstract}

(C) 2017 Sharif University of Technology. All rights reserved.

\section{Introduction}

Process monitoring is an essential activity for the improvement of product and service quality. All the processes experience variations in their outputs, and quality is affected by them. These variations are mainly classified into two types: natural and unnatural. The natural variations are inherent parts of any process, and these are not harmful to the process. The unnatural variations deteriorate the process quality and lead us, generally, to unusual patterns in

*. Corresponding author. Tel.: 00923235332733

E-mail addresses: saddamabbasi@yahoo.com (S.A. Abbasi); shabbirahmadr86@yahoo.com (S. Ahmad);

riazm@kfupm.edu.sa (M.Riaz) the process behavior. We may live with the natural variations in a process, while the unnatural variations need timely detection and corrective actions. Control charts are very popular tools used in the monitoring of manufacturing and non-manufacturing processes. We are generally concerned with the stability of the parameters of the quality characteristic(s) of interest such as location and dispersion. There are two main types of control charts, namely memory-less (Shewhart) and memory (Exponentially Weighted Moving Average control charts (EWMA) and cumulative sum (CUSUM)). The Shewhart type charts are meant for larger shifts, while EWMA and CUSUM type charts deal with the smaller shifts ([1-3]). Under the ideal conditions, we assume normality of the quality characteristic(s) of interest for the traditional 
design structures of different types of control charts. In practice, normality assumption is quite stringent, and we may not be able to fulfill it for many variables of interest in different processes. This necessitates the use of some alternative procedures that do not depend on this hard to meet assumption of normality.

Nonparametric control charts are designed for the reason mentioned above, where we are free from the distributional assumptions. An inappropriate use of parametric control charts, instead of nonparametric charts, may lead us to unfavorable outcomes in the form of detection ability and high false alarm rates. Therefore, in the absence of strict distributional assumptions, it is always better to use the nonparametric control charts. We may find a variety of literature including some recent contributions in the direction of nonparametric control charts such as [4-14], among many others.

In the direction of nonparametric memory-less charts, we may find different proposals in the form of nonparametric EWMA (NPEWMA) and nonparametric CUSUM (NPCUSUM) control charts. Li et al. [15] used Man-Whitney statistics and suggested NPEWMA and NPCUSUM charts. Zou and Tsung [16] proposed a multivariate EWMA control chart using the weighted version of the sign statistic. Graham et al. [17] proposed NPEWMA sign chart for monitoring process location using individual observations. Yang et al. [18] proposed two NPEWMA control charts, namely the nonparametric EWMA sign and the nonparametric Arcsine EWMA sign charts, for quick detection of shifts from process target using the well-known sign statistic. They [18] used the asymptotic control limits due to the ease of computation for NPEWMA charts. This makes the NPEWMA chart insensitive to startup quality problems. When the process is initially outof-control, it is important to detect the sources of these out-of-control conditions as early as possible so that corrective actions may be taken at an early stage. The time-varying structure of the limits helps resolve this issue. Moreover, the sensitivity of timevarying NPEWMA chart can be increased further at process startup using Fast Initial Response (FIR) feature. This study investigates the performance of NPEWMA charts using asymptotic, time-varying, and two variants of FIR based control limits. For the said purposes, we used the nonparametric Arcsine EWMA sign chart proposed by Yang et al. [18]. We will refer to this asymptotic control limits-based chart as NPAS chart for the rest of this study. The time-varying structure will be referred to by TNPAS using timevarying feature given in [19]. We will also consider two FIR-based control structures, namely FNPAS chart using FIR feature of [20] and IFNPAS chart using FIR feature of [21]. The rest of the study is organized as follows. Section 2 introduces the structure of the NPAS chart and further presents the designs of TNPAS, FNPAS, and IFNPAS charts. Section 3 evaluates run length characteristics of different charting structures under study and provides a comparative analysis. Section 4 provides an application example for illustration purposes. Section 5 gives a summary and the concluding remarks of the study.

\section{Design structures of NPEWMA control charts}

This section provides the structure of NPAS chart and further extends its design by using time-varying and fast initial response features in the form of three charts, namely TNPAS, FNPAS, and IFNPAS charts. Before defining these structures, we first provide description of some necessary terms and symbols below.

Let $X_{1}, X_{2}, \ldots, X_{n}$ be a random sample of size $n$ drawn from a process with $\mu$ as its location parameter. Let us define a transformed variable $Y$ as $Y_{i}=X_{i}-\mu$. Suppose that $p$ is a probability measure defined as $p=\operatorname{Pr}\left(Y_{i}>0\right)$ which takes an in-control value of $p_{0}$ and otherwise out-of-control. It is to be mentioned that we will be monitoring the location parameter by assessing the stability of $p$ with reference to its incontrol value $p_{0}$. Let $I$ be an indicator variable defined as $I=1$ if $Y_{i}>0$ and zero otherwise. Based on this indicator variable, we have $M=\sum_{i=1}^{n} I_{i}$, where $M$ follows binomial distribution with parameters $n$ and $p_{0}$ (cf. [18]).

Using the above-defined variable $M$, the arcsine transformation and the approximate distribution are provided as (cf. [18]):

$$
Z=\sin ^{-1} \sqrt{M / n} \sim N\left(\sin ^{-1} \sqrt{p_{0}}, 1 / 4 n\right) .
$$

The EWMA statistic for $Z$ is defined as: $W_{t}=\lambda Z_{t}+$ $(1-\lambda) W_{t-1}$, where $\lambda$ is the smoothing constant $(0<$ $\lambda \leq 1)$ and $W_{0}=\sin ^{-1} \sqrt{p_{0}}$.

Based on the EWMA statistic $W_{t}$, Yang et al. [18] introduced a nonparametric EWMA control chart (we named it as the NPAS chart). The control limits (Upper Control Limit (UCL) and Lower Control Limit (LCL)) for NPAS chart are given as (cf. [18]):

\section{NPAS Chart}

$$
\left.\begin{array}{l}
\mathrm{UCL}=\sin ^{-1} \sqrt{p_{0}}+L \sqrt{(\lambda /(2-\lambda))(1 / 4 n)} \\
\mathrm{LCL}=\sin ^{-1} \sqrt{p_{0}}-L \sqrt{(\lambda /(2-\lambda))(1 / 4 n)}
\end{array}\right\}
$$

where $L$ represents the control limits multiplier that depends on $n$ and $\lambda$. It is to be mentioned that the Central Line (CL) is defined as $\mathrm{CL}=\sin ^{-1} \sqrt{p_{0}}$ for NPAS charts (and all the other charts under discussion in this study). The statistic $W_{t}$ is used as plotting 


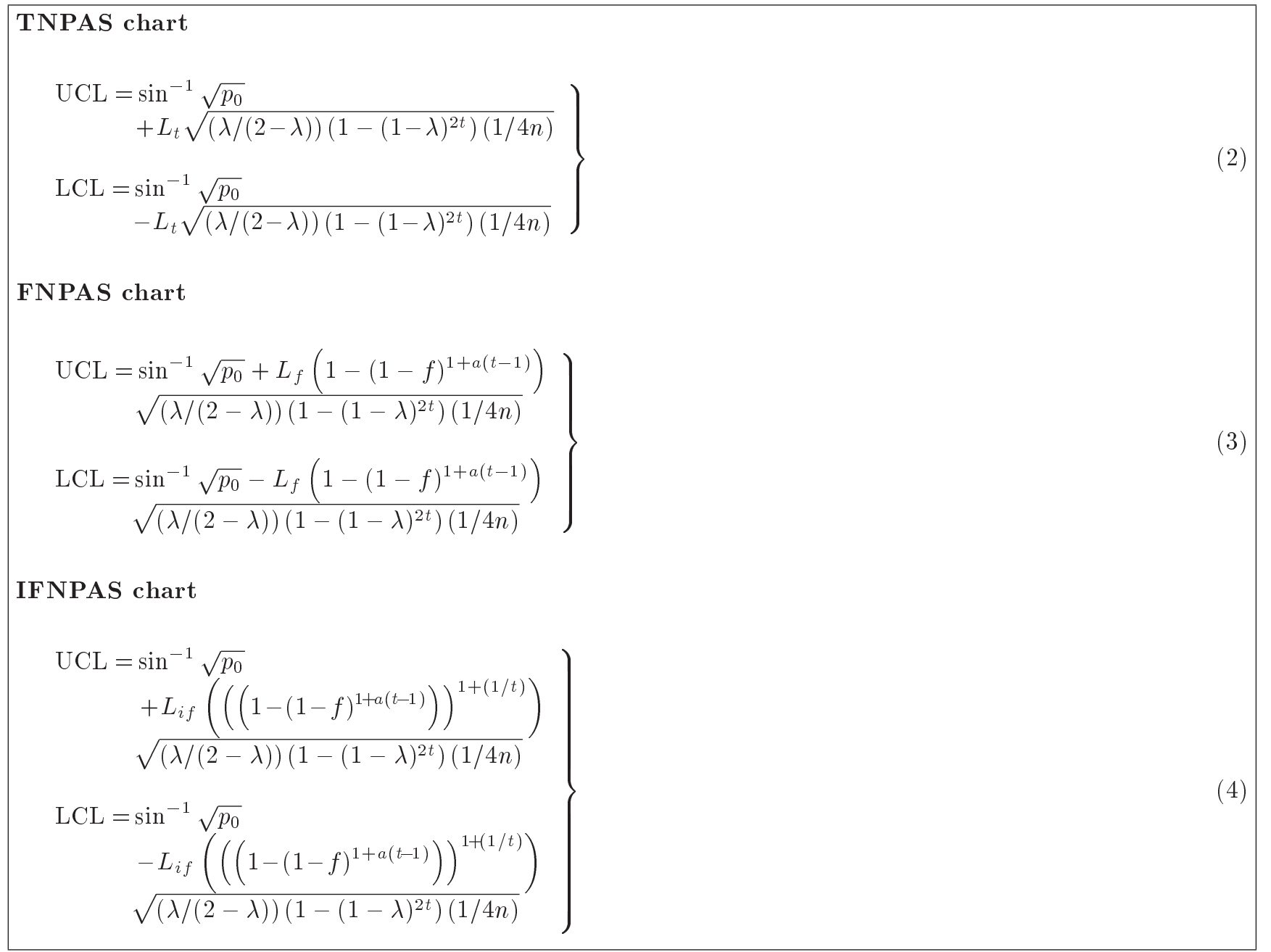

Box I

statistics against these control limits in the form of UCL, LCL, and CL.

We intend to extend here the design structure of NPAS chart using time-varying and fast initial response features. The time-varying structure will be referred to by TNPAS, while FNPAS and IFNPAS further add the usual and an improved FIR features, respectively, in rest of this study. Previous studies showed that the use of time-varying control limits, as compared to asymptotic limits, significantly improves the out-of-control run length behavior of the NPEWMA control chart. A further increase in the sensitivity of NPEWMA chart to detect shifts in process target can be achieved by an FIR feature. The FIR feature, introduced by Lucas and Crosier [22] for CUSUM charts, detects outof-control signals more quickly at process startup by assigning some non-zero constant to the starting values of CUSUM chart statistics. Lucas and Saccucci [23] proposed the idea of applying the FIR feature to EWMA control structures by two one-sided EWMA charts. Rhoads et al. [24] used the FIR approach for time-varying control limits and showed superior performance of their proposed scheme compared to the [23] FIR Scheme. Both of these schemes were criticized as they required the use of two EWMA charts instead of one for monitoring changes in process parameters. Steiner [20] presented another FIR scheme for EWMA charts that is based on further narrowing the time-varying control limits by an exponentially decreasing FIR adjustment. Furthermore, Haq et al. [21] suggested an improvement over the FIR scheme of [20]. The description of the three said charts is given in Box I, where $a$ is known as the adjustment parameter and is chosen such that the FIR adjustment has very little effect after a specified time period (say at $t=20$, we have $\mathrm{FIR}_{\mathrm{adj}}=0.99$. To obtain a substantial benefit from FIR feature, $f$ should be fairly small. In this study, we used $f=0.5$ and limited the effect of FIR adjustment till $t=20$ following [20,25].

It is to be noted that the design structures of TNPAS and FNPAS charts given in Eqs. (2) and (3), respectively, may be linked with each other by a mathematical relationship. For example, if we consider the UCLs of the two structures, we may observe the 
following relationship:

$\mathrm{UCL}_{\mathrm{FNPAS}}=\mathrm{UCL}_{\mathrm{TNPAS}}$

$$
\begin{aligned}
& +\sqrt{(\lambda /(2-\lambda))\left(1-(1-\lambda)^{2 t}\right)(1 / 4 n)} \\
& {\left[L_{f}(1-(1-f))^{1+a(t-1)}-L_{t}\right] .}
\end{aligned}
$$

We may also replace $\left[L_{f}(1-(1-f))^{1+a(t-1)}-L_{t}\right]$ with $L_{u}$ to get:

$$
\begin{aligned}
\mathrm{UCL}_{\mathrm{FNPAS}}= & \mathrm{UCL}_{\mathrm{TNPAS}} \\
& +L_{u} \sqrt{(\lambda /(2-\lambda))\left(1-(1-\lambda)^{2 t}\right)(1 / 4 n)} .
\end{aligned}
$$

In addition to the above relationship given in Eqs. (5) and (6), one may also observe the asymptotic relationship of the design structures of IFNPAS charts (given in Eq. (4), respectively) with the design structure of other charts.

\section{Performance evaluation and comparisons}

In this section, the control charts under study are compared with each other in terms of different measures including Average Run Length (ARL), median run length (MDRL), Standard Deviation Run Length (SDRL), Extra Quadratic Loss (EQL), Relative Average Run Length (RARL), and Performance Comparison Index (PCI). The measures ARL, MDRL, and SDRL are obtained from the distribution of Run Length (RL) at each shift value. On the other hand, EQL and RARL are calculated over the entire shift range to explore overall effectiveness of a control structure, while PCI is obtained from EQL values. Usually, two ARL values, namely $\mathrm{ARL}_{0}$ and $\mathrm{ARL}_{1}$, are used for performance evaluation. $\mathrm{ARL}_{0}$ is the average number of samples from an in-control process until an outof-control signal is detected by a control chart, while $\mathrm{ARL}_{1}$ is the average number of samples until an outof-control signal is detected by a control chart when the process is shifted to out-of-control scenario. It is usually preferable to get large values of $\mathrm{ARL}_{0}$ and small values of $\mathrm{ARL}_{1}$ for any control chart setting. It is also expected to get smaller value of MDRL and SDRL for an efficient control chart. The measures EQL, RARL, and PCI can be defined mathematically as:

$$
\begin{aligned}
& \mathrm{EQL}=\frac{1}{\delta_{\max }-\delta_{\min }} \int_{\delta_{\text {min }}}^{\delta_{\max }} \delta^{2} \operatorname{ARL}(\delta) d \delta, \\
& \mathrm{RARL}=\frac{1}{\delta_{\max }-\delta_{\min }} \int_{\delta_{\text {min }}}^{\delta_{\max }} \frac{\operatorname{ARL}(\delta)}{\operatorname{ARL}_{\mathrm{opt}}(\delta)} d \delta,
\end{aligned}
$$

$$
\mathrm{PCI}=\frac{\mathrm{EQL}}{\mathrm{EQL}_{\mathrm{opt}}}
$$

where $\delta$ is the amount of shift in the process mean $\mu_{y}$. The EQL is a weighted average ARL over the whole process shift domain $\delta_{\min }<\delta<\delta_{\max }$ using the square of shift $\left(\delta^{2}\right)$ as a weight. It is to be mentioned that a chart with minimum EQL value is considered to be an optimum chart. The measure RARL describes the overall effectiveness of a particular chart relative to the optimum chart, where $\operatorname{ARL}(\delta)$ and $\operatorname{ARL}_{\text {opt }}(\delta)$ are the average run lengths of the particular and optimum charts at $\delta$, respectively. The RARL $=1$ may be observed for optimum chart, while RARL $>1$ is for other charts which shows that the particular chart exhibits inferior performance relative to the optimum chart. The PCI is the ratio between the EQL values of the particular chart and the optimum chart. PCI $=1$ may be observed for the chart with minimum EQL value, while PCI $>1$ for other charts. Some of the researchers used different versions of the above measures in their studies; for instance [26-36]. As both RARL and PCI usually result in similar relative performance of the charts (cf. [30]), hence, we are only concentrating on RARL for the rest of the study.

For the computation of these measures, we have used Monte Carlo simulations (for ARL) and numerical integration (for the other measures). The computational algorithm for these measures is given as: i) Generate random samples from any probability model; ii) Compute the sample statistics; iii) Set the control limits using the description given in Section 2; iv) Use steps (i)-(iii) to implement the procedural steps of ARL depending on the choices of and control chart multipliers $\left(L, L_{t}, L_{f}, L_{i f}\right)$ as defined above in Section 2 (this results in ARL values); v) Based on the results of step (iv) for ARL as a function of $\delta$, multiply ARL, divide range of values, and then integrate the output over the entire range using an appropriate numerical integration technique (like Simpson or Trapezoidal) (this results in EQL value); vi) Repeat steps (iv) and (v) for all the charts; and vii) Based on the results of step (vi), take the ratio of the ARL of a particular chart by the ARL of the benchmark chart (the usual one in this study), divide by the range of values, and then integrate the output over the entire range using an appropriate numerical integration technique (like Simpson or Trapezoidal) (this results into RARL value).

It is to be mentioned that the number of values determines the number of subintervals used by the numerical integration technique and with the increase in this number, one may obtain more precise results for these measures. For our study purposes, we have used Simpson's method of numerical integration. By varying the values of control chart parameters, ARLs are calculated 
Table 1. Run length characteristics of NPAS chart for different values of $n$ and $p$ when $\mathrm{ARL}_{0}=370$ and $\lambda=0.05$ and 0.25 .

\begin{tabular}{|c|c|c|c|c|c|c|c|c|c|c|c|c|c|c|c|}
\hline \multirow[b]{2}{*}{$\lambda$} & \multirow[b]{2}{*}{$n$} & & \multicolumn{13}{|c|}{$P$} \\
\hline & & & 0.05 & 0.20 & 0.30 & 0.35 & 0.40 & 0.45 & 0.50 & 0.55 & 0.60 & 0.65 & 0.70 & 0.80 & 0.9 \\
\hline \multirow{12}{*}{0.05} & 5 & ARL & 31 & 14 & 8 & 1 & 32.47 & 5 & 3 & 8 & 4 & 9 & 0 & 1 & \\
\hline & & MDRL & 4.00 & 7.00 & 11.00 & 16.00 & 28.00 & 68.00 & 256.00 & 68.00 & 27.00 & 16.00 & 11.00 & 7.00 & 4.00 \\
\hline & & SDRL & 0.88 & 2.34 & 5.17 & 8.85 & 20.23 & 74.05 & 363.54 & 77.15 & 19.97 & 8.86 & .15 & 2.32 & 0.89 \\
\hline & 7 & $\triangle \mathrm{RI}$ & 3.30 & 5.82 & 9.8 & 14.36 & 25.04 & 70.38 & 372.60 & 70.13 & 25.09 & 14.33 & .78 & 5.83 & 1 \\
\hline & & MDRL & 3.00 & 6.00 & 9.00 & 13.00 & 22.00 & 54.00 & 263.00 & 54.00 & 22.00 & 13.00 & .00 & 6.00 & 3.00 \\
\hline & & SDRL & 0.54 & 1.73 & 3.68 & 6.40 & 14.19 & 56.34 & 360.39 & 55.18 & 14.27 & 6.51 & 3.69 & 1.73 & 0.55 \\
\hline & 10 & ARL & 2.71 & 4.78 & 7.90 & 11.26 & 19.12 & 52.54 & 372.33 & 52.56 & 19.23 & 11.23 & 7.88 & 4.79 & 271 \\
\hline & & MI & 3.00 & 5.00 & 8.0 & 10.00 & 17.00 & 0 & 264 & 0 & 17.00 & 1 & 0 & 5.00 & 2 \\
\hline & & SDRL & 0.59 & 1.29 & 2.63 & 4.48 & 65 & 38.71 & 354.41 & 38.27 & U & 4. & 61 & 1.29 & 0 \\
\hline & 15 & ARL & 2.31 & 3.97 & 6.32 & 8.88 & 14.62 & 38.84 & 369.02 & 38.37 & 14.65 & 8.85 & 6.31 & 3.94 & 2.31 \\
\hline & & MDRL & 2.00 & 4.00 & 6 & 0 & $1:$ & 32 & 260 & 0 & 13 & 8 & 0 & 4.00 & 2.00 \\
\hline & & $\mathrm{S}$ & 0.47 & 0.94 & ? & 3.04 & 6.50 & 26.05 & 0 & 9 & & 3.04 & 1 & 3 & 0.46 \\
\hline \multirow{12}{*}{ 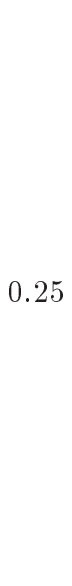 } & 5 & ARL & 2.58 & 6.07 & 14.12 & 26.21 & 58.78 & 174.46 & 369.03 & 170.97 & 60.30 & 25.94 & 13.90 & 5.91 & 2.52 \\
\hline & & MDRL & 2.00 & 5.00 & 11.00 & 20.00 & 43.00 & 121.00 & 255.00 & 121.00 & 43.00 & 19.00 & 11.00 & 6.17 & 2. \\
\hline & & SDRL & 0.85 & 3.40 & 10.46 & 21.87 & 53.41 & 171 & 370.82 & 16 & 56 & 2 & 10 & 5.00 & 2.00 \\
\hline & 7 & AI & 2. & 4.89 & 10 & 2 & 4 & $1:$ & 36 & $\$ 1$ & 6 & 5 & 10.99 & 3.49 & 9 \\
\hline & & MDRL & 2.00 & 4.00 & 9.00 & 15.00 & 34.00 & 106.00 & 254.00 & 105.00 & 34.00 & 16.00 & 9.00 & 4.86 & 2.40 \\
\hline & & SDRL & 0.60 & 2.43 & 7.55 & 16.34 & 43.43 & 146.56 & 367.95 & 145.83 & 43.30 & 16.58 & 7.70 & 4.00 & 2.00 \\
\hline & 10 & ARL & 1.47 & 3.42 & 6.99 & 12.37 & 29.13 & 109.55 & 368.93 & 110.84 & 29.65 & 12. & 7.02 & 2.42 & 1 \\
\hline & & MDRL & 1.00 & 3.00 & 6.00 & 10.00 & 22.00 & 76.00 & 257.00 & 79.00 & 22.00 & 10.00 & 6.00 & 3.40 & 1. \\
\hline & & SDRL & 0.61 & 1.56 & 4.04 & 8.80 & 25.13 & 106.10 & 367.92 & 107.20 & 25.41 & 8.98 & 4.07 & 3.00 & 1.00 \\
\hline & 15 & ARL & 1.53 & 2.71 & 4.8 & 7.98 & 17.03 & 69.72 & 373.21 & 70.04 & 17.36 & 7.97 & 4.87 & 1.56 & 1.13 \\
\hline & & MDRL & 2.00 & 3.00 & 4.00 & 7.00 & 13.00 & 49.00 & 256.50 & 50.00 & 14.00 & 7.00 & 4.00 & 2.71 & 1.55 \\
\hline & & SDRL & 0.51 & 0.89 & 2.25 & 4.72 & 13.09 & 67.03 & 377.22 & 64.80 & 13.34 & 4.75 & 2.26 & 3.00 & 2.00 \\
\hline
\end{tabular}

for all the charts, followed by the computations of EQL and RARL using the algorithm provided above. In order to obtain these measures, 10,000 repetitions are used that provide the results with satisfactory relative standard errors (c.f. [23,37-39]). It is to be mentioned that the precision of the results may be increased by increasing the number of simulations, however, up to 5000 simulations may serve the purpose quite effectively, as may be seen in the literature such as $[40,41]$.

In order to see the impact of smoothing parameter $\lambda$, the performance of NPAS, TNPAS, FNPAS, and IFNPAS charting schemes are investigated using the above performance measures for $\lambda=0.05$ and 0.25 . The summary of the run length characteristics including ARL, MDRL and SDRL of NPAS, TNPAS, FNPAS, and IFNPAS charts is reported in Tables 14 , respectively, for $\lambda=0.05$ and 0.25 . The relative standard errors of the results are found to be around $1.5 \%$ as checked by repeating the simulations. This is quite acceptable in control chart studies (for details, see $[40,41])$. The columns, corresponding to $p=p_{0}=$ 0.50 in Tables $1-4$, provide the run length character- istics of four charts when the process is assumed to be in statistical control. The process is said to be out-of-control for $p \neq 0.50$. Control chart multiples $L, L_{t}, L_{f}$ are $L_{i f}$ also chosen as to give the same in-control average run length of 370 (i.e., $\mathrm{ARL}_{0}=$ $370)$ to NPAS, TNPAS, FNPAS, and IFNPAS charts, respectively.

\subsection{Comparison of run length characteristics of NPAS and TNPAS charts}

In this section, we evaluate the performance of NPAS and TNPAS charts using the performance measures discussed earlier. The ARL results for the NPAS chart are in close agreement with the results reported in [18], showing the accuracy of our simulation routines. [18] investigated the ARL behavior of his proposed NPAS chart only for $\lambda=0.05$. The results in Tables 1 and 2 indicate that for small values of $\lambda$ (which is most popular choice of EWMA charts), the $\mathrm{ARL}_{1}$ of the TNPAS chart is significantly lower than the $\mathrm{ARL}_{1}$ of NPAS chart, see, for example, when $n=10$ and $\lambda=0.05$, the $\mathrm{ARL}_{1}=47.30,14.54$ and 7.31 for TNPAS 
Table 2. Run length characteristics of TNPAS chart for different values of $n$ and $p$ when $\mathrm{ARL}_{0}=370$ and $\lambda=0.05$ and 0.25 .

\begin{tabular}{|c|c|c|c|c|c|c|c|c|c|c|c|c|c|c|c|}
\hline \multirow[b]{2}{*}{$\lambda$} & \multirow[b]{2}{*}{$n$} & & \multicolumn{13}{|c|}{$\boldsymbol{P}$} \\
\hline & & & 0.05 & 0.20 & 0.30 & 0.35 & 0.40 & 45 & 0.50 & 0.55 & 0.60 & 0.65 & .70 & 0.80 & 0.95 \\
\hline \multirow{12}{*}{0.05} & 5 & ARL & 1.32 & 3.44 & 7.66 & 12.77 & 26.15 & 83.15 & 370.58 & 82.93 & 26.11 & 13.01 & 7.80 & 3.49 & 1.32 \\
\hline & & MDRL & 1.00 & 3.00 & 7.00 & 11.00 & 21.00 & 59.00 & 241.00 & 60.00 & 21.00 & 11.00 & 7.00 & 3.00 & 1.00 \\
\hline & & SDRL & 0.68 & 2.55 & 5.89 & 10.17 & 22.48 & 81.62 & 401.11 & 81.23 & 22.51 & 10.36 & 5.96 & 2.60 & 067 \\
\hline & 7 & ARL & 1.33 & 2.94 & 6.08 & 10.05 & 20.04 & 63.50 & 370.51 & 63.87 & 20.25 & 10.10 & .10 & 2.94 & 33 \\
\hline & & MDRL & 1.00 & 2.00 & 5.00 & 9.00 & 16.00 & 47.00 & 251.00 & 48.00 & 17.00 & 9.00 & 5.00 & 2.00 & 1.00 \\
\hline & & SDRL & 0.53 & 1.77 & 4.09 & 7.08 & 15.57 & 59.18 & 388.69 & 59.10 & 15.74 & 7.15 & 4.06 & 1.77 & 0.53 \\
\hline & 10 & ARL & 1.08 & 2.17 & 4.49 & 7.31 & 14.57 & 46.96 & 369 & 47.30 & 14.54 & 7.31 & 4.47 & 2.17 & 1.09 \\
\hline & & MDRL & 1.00 & 2.00 & 4.00 & 6.00 & 12.00 & 36.00 & 247.00 & 37.00 & 12.00 & 6.00 & 1.00 & 2.00 & $\pi 0$ \\
\hline & & SDRL & 0.28 & 1.24 & 2.87 & 5.01 & 10.83 & 40.98 & 386.56 & 41.18 & 10.78 & 5.00 & 2.82 & 1.24 & 0.29 \\
\hline & 15 & ARL & 1.04 & 1.77 & 3.21 & 5.22 & 10.32 & 33.20 & 369.44 & 33.28 & 10.33 & 5.25 & 3.25 & 1.75 & 1.04 \\
\hline & & MDRL & 1.00 & 2.00 & 3.00 & 1.00 & .00 & 27.00 & 246.00 & 27.00 & 9.00 & 4.00 & .00 & 00 & 0 \\
\hline & & SDRL & 0.19 & 0.76 & 1.80 & 3.33 & 7.23 & 27.19 & 388.63 & 26.97 & 7.20 & 3.36 & .84 & 0.75 & U \\
\hline \multirow{12}{*}{0.25} & 5 & ARL & 2.53 & 5.77 & 13.66 & 26.23 & 60.51 & 175.59 & 370.24 & 173.38 & 59.31 & 26.05 & 13.66 & 5.79 & 2.52 \\
\hline & & MDRL & 2.00 & 5.00 & 11.00 & 20.00 & 43.00 & 123.00 & 254.00 & 119.00 & 43.00 & 19.00 & 11.00 & 5.00 & 2.00 \\
\hline & & SDRL & 0.77 & 3. & 1 & 2 & 5 & 17 & 3 & 1 & 3 & 6 & 51 & 19 & 0 \\
\hline & 7 & ARL & 1.42 & 4.05 & 10.02 & 18.93 & 46.16 & 147.45 & 369.76 & 149.04 & 46.83 & 19.19 & .86 & 4.01 & 1.43 \\
\hline & & MDRL & 1.00 & 4.00 & 8.00 & 14.00 & 33.00 & 104.00 & 256.00 & 103.50 & 33.00 & 15.00 & 8.00 & 4.00 & 1.00 \\
\hline & & SDRL & 0.74 & 2.73 & 8.05 & 16.57 & 44.54 & 146.75 & 369.54 & 149.25 & 44.59 & 16.83 & 7.77 & 2.66 & 0.75 \\
\hline & 10 & ARL & 1.42 & 3.04 & 6.51 & 11.71 & 28.49 & 110.14 & 370.71 & 111.43 & 28.72 & 11.91 & 6.55 & 3.03 & 1 \\
\hline & & MDRL & 1.00 & 3.00 & 6.00 & 10.00 & 21.00 & 78.00 & 255.00 & 78.00 & 21.00 & 10.00 & 6.00 & 3.00 & 1.00 \\
\hline & & SDRL & 0.52 & 1.50 & 4.15 & 8.54 & 25.22 & 104.99 & 369.52 & 109.17 & 25.52 & 8.90 & 4.18 & 1.49 & 0.52 \\
\hline & 15 & ARL & 1.04 & 1.95 & 4.13 & 7.11 & 16.47 & 68.80 & 370.08 & 70.68 & 16.36 & 7.18 & 4.10 & 1.96 & 1.04 \\
\hline & & MDRL & 1.00 & 2.00 & 4.00 & 6.00 & 13.00 & 48.00 & 255.00 & 50.00 & 13.00 & 6.00 & 4.00 & 2.00 & 1.00 \\
\hline & & SDRL & 0.19 & 1.00 & 2.48 & 4.88 & 13.67 & 66.73 & 381.88 & 67.43 & 13.45 & 4.93 & 2.44 & 1.01 & 0.19 \\
\hline
\end{tabular}

chart at $p=0.55,0.60$ and 0.65 , respectively, while for NPAS chart, the $\mathrm{ARL}_{1}=52.56,19.23$ and 11.23 for the same values of $n, \lambda$, and $p$. It indicates that TNPAS chart requires on average nearly five less samples as compared to NPAS chart to detect a shift in process target when $\lambda=0.05$. Moreover, MDRL of TNPAS chart is also lower than MDRL of NPAS chart, while there is a slight increase in SDRL of the TNPAS chart as compared to NPAS chart. The ARL curves of NPAS and TNPAS charts along with other charts will be compared graphically in the next section.

\subsection{Effect of fast initial response on NPEWMA chart}

We have seen in the previous section that the use of time-varying control limits as compared to asymptotic limits significantly improved the out-of-control run length behavior of the NPEWMA control chart. In this section, we examine the effect of FIR on the performance of NPEWMA chart. The run length characteristics of FNPAS and IFNPAS charts are reported in Tables 3 and 4, respectively. By comparing the results, we can observe the superior run length performance of the IFNPAS chart as compared to the FNPAS chart, respectively. For example, the $\mathrm{ARL}_{1}=$ $31.62,7.96$ and 3.40 for IFNPAS chart at $p=0.55$, 0.60 and 0.65 , while for FNPAS chart, the $\mathrm{ARL}_{1}=$ $39.02,10.80$ and 4.79 using $n=10$ and $\lambda=0.05$ while the corresponding $\mathrm{ARL}_{1}$ values from Tables 1 and 2 exhibit the inferior performance of TNPAS and NPAS charts, respectively, as compared to IFNPAS and FNPAS charts. This indicates that the FNPAS chart requires on average nearly 8,4 and 3 less samples as compared to TNPAS chart and 13, 9, 7 less samples as compared to the NPAS chart to detect departures from $\lambda=0.05$ to $p=0.55,0.60$, and 0.65 . On the other hand, the IFNPAS chart requires on average nearly 8 , 3 , and 1 samples as compared to FNPAS chart; 16, 7, and 4 samples as compared to TNPAS chart; 21, 12, and 8 samples as compared to NPAS chart. Moreover, MDRL of IFNPAS chart is also lower than MDRL of FNPAS chart followed by TNPAS and NPAS charts, while there is slight increase in SDRL of these charts with the same order. 
Table 3. Run length characteristics of FNPAS chart for different values of $n$ and $p$ when $\mathrm{ARL}_{0}=370$ and $\lambda=0.05$ and 0.25 .

\begin{tabular}{|c|c|c|c|c|c|c|c|c|c|c|c|c|c|c|c|}
\hline \multirow[b]{2}{*}{$\lambda$} & \multirow[b]{2}{*}{$n$} & & \multicolumn{13}{|c|}{$\boldsymbol{P}$} \\
\hline & & & 0.05 & 0.20 & 0.30 & 0.35 & 0.40 & 0.45 & 0.50 & 0.55 & 0.60 & 0.65 & 0.70 & 0.80 & 0.95 \\
\hline \multirow{12}{*}{0.05} & 5 & ARL & 1.25 & 2.52 & 5.67 & 10.21 & 22.14 & 75.18 & 371.19 & 76.31 & 22.77 & 10.30 & 5.60 & 2.50 & 1.24 \\
\hline & & MDRL & 1.00 & 2.00 & 3.00 & 6.00 & 15.00 & 49.00 & 211.00 & 49.00 & 16.00 & 6.00 & 3.00 & 2.00 & 1.00 \\
\hline & & SDRL & 0.46 & 1.94 & 5.55 & 10.47 & 23.55 & 86.74 & 467.12 & 89.38 & 23.96 & 10.57 & 5.57 & 1.95 & 0.47 \\
\hline & 7 & ARL & 1.05 & 1.77 & 3.83 & 6.99 & 15.34 & 54.71 & 370.30 & 54.56 & 15.34 & 6.85 & 3.79 & 1.76 & 1.05 \\
\hline & & MDRL & 1.00 & 1.00 & 2.00 & 4.00 & 9.00 & 35.00 & 203.00 & 35.00 & 9.00 & 3.00 & 2.00 & 1.00 & 1.00 \\
\hline & & SDRL & 0.23 & 1.24 & 3.83 & 7.39 & 16.64 & 64.63 & 473.06 & 63.61 & 16.59 & 7.39 & 3.69 & 1.23 & 0.23 \\
\hline & 10 & ARL & 1.01 & 1.43 & 2.71 & 4.77 & 10.71 & 39.25 & 369.82 & 39.02 & 10.80 & 4.79 & 2.70 & 1.42 & 1.01 \\
\hline & & MDRL & 1.00 & 1.00 & 2.00 & 3.00 & 6.00 & 26.00 & 200.00 & 25.00 & 6.00 & 3.00 & 2.00 & 1.00 & 1.00 \\
\hline & & SDRL & 0.10 & 0.75 & 2.38 & 4.83 & 11.36 & 44.28 & 466.38 & 45.09 & 11.53 & 4.77 & 2.40 & 0.72 & 0.11 \\
\hline & 15 & ARL & 1.00 & 1.18 & 1.94 & 3.19 & 7.20 & 27.00 & 370.04 & 26.43 & 7.25 & 3.21 & 1.95 & 1.19 & 1.00 \\
\hline & & MDRL & 1.00 & 1.00 & 1.00 & 2.00 & 7.00 & 18.00 & 199.00 & 18.00 & 4.00 & 2.00 & 1.00 & 1.00 & 1.00 \\
\hline & & SDRL & 0.02 & 0.45 & 1.41 & 3.01 & 7.47 & 29.81 & 466.66 & 29.15 & 7.50 & 2.97 & 1.39 & 0.45 & 0.02 \\
\hline \multirow{12}{*}{0.25} & 5 & ARL & 1.28 & 3.27 & 8.86 & 18.61 & 49.16 & 158.61 & 370.17 & 163.82 & 48.93 & 18.17 & 8.59 & 3.24 & 1.29 \\
\hline & & MDRL & 1.00 & 2.00 & 5.00 & 10.00 & 27.00 & 95.00 & 227.00 & 100.00 & 27.00 & 9.00 & 5.00 & 2.00 & 1.00 \\
\hline & & SDRL & 0.60 & 2.94 & 10.32 & 22 . & 6 & 18 & 43 & 1. & 7 & 22 & 9.70 & 2.87 & 0.62 \\
\hline & 7 & ARL & 1.05 & 2.00 & 5.60 & 12.48 & 35.85 & 136.47 & 370.91 & 136.54 & 35.76 & 12.26 & 5.56 & 1.98 & 1.04 \\
\hline & & MDRL & 1.00 & 1.00 & 3.00 & 6.00 & 18.00 & 77.00 & 220.00 & 74.00 & 18.00 & 6.00 & 3.00 & 1.00 & 1.00 \\
\hline & & SDRL & 0.23 & 1.67 & 6.82 & 16.27 & 46.42 & 169.75 & 464.29 & 174.97 & 46.29 & 15.94 & 6.86 & 1.71 & 0.22 \\
\hline & 10 & ARL & 1.01 & 1.49 & 3.27 & 6.69 & 20.41 & 100.58 & 369.96 & 99.25 & 20.39 & 6.72 & 3.28 & 1.50 & 1.01 \\
\hline & & MDRL & 1.00 & 1.00 & 2.00 & 4.00 & 10.00 & 56.00 & 213.00 & 55.00 & 9.00 & 4.00 & 2.00 & 1.00 & 1.00 \\
\hline & & SDRL & 0.12 & 0.90 & 3.30 & 8.05 & 26.92 & 125.21 & 448.51 & 123.59 & 26.64 & 8.04 & 3.33 & 0.92 & 0.10 \\
\hline & 15 & ARL & 1.00 & 1.20 & 2.10 & 3.75 & 10.16 & 57.47 & 370.49 & 57.28 & 10.06 & 3.74 & 2.11 & 1.20 & 1.00 \\
\hline & & MDRL & 1.00 & 1.00 & 1.00 & 2.00 & 5.00 & 29.00 & 218.00 & 28.00 & 5.00 & 2.00 & 1.00 & 1.00 & 1.00 \\
\hline & & SDRL & 0.02 & 0.51 & 1.66 & 3.89 & 12.72 & 75.33 & 461.12 & 75.68 & 13.22 & 3.93 & 1.71 & 0.51 & 0.02 \\
\hline
\end{tabular}

The performances of NPAS, TNPAS, FNPAS, and IFNPAS charts are also evaluated graphically by creating ARL curves, provided in Figures 1 and 2, for the same values of $n, p, \mathrm{ARL}_{0}$, and $\lambda$ as discussed in Tables 1-4. It can be observed from Figures 1 and 2 that the difference in the ARL curves of NPAS and TNPAS charts is larger for small value of $\lambda$ (i.e., $\lambda=$ $0.05)$. As the value of $\lambda$ increases to 0.25 , the difference seems to be smaller. For $\lambda=1$, we expect to see a similar performance of these two charts. The benefit of using the FIR feature can be easily seen here as the ARL curves of the FNPAS chart are consistently lower than the ARL curves of both NPAS and TNPAS charts, while the ARL curve of IFNPAS chart lies lower than FNPAS chart for every choice of $n, p$, and $\lambda$ in general. Moreover, from EQL and RARL values provided in Table 5 , it is indicated that for every choice of $n, p$, and $\lambda$, the EQL values of IFNPAS chart are smaller than those of FNPAS chart followed by TNPAS and NPAS charts in general. Moreover, the values of RARL for IFNPAS chart are equal to 1 , while these values are greater than 1 for other charts, justifing the superiority of IFNPAS chart over other charts. This indicates that the FIR-based NPEWMA charts detect shifts in process target more quickly as compared to NPEWMA charts based on asymptotic or time-varying control limits.

\subsection{Investigation of some additional features}

In this subsection, we provide some additional investigations about different features of NPAS, TNPAS, FNPAS, and IFNPAS charts. These include the following: control chart coefficients at different $\mathrm{ARL}_{0}$ values; run length characteristics of EWMA charts using FIR without the time varying feature; the effect of parameter $f$ on the performance of EWMA chart using FIR feature; and percentile points of the run length distribution for in-depth analysis.

Control chart coefficients at different $\mathbf{A R L}_{0}$ values: We have considered $A R L_{0}=370$ for the performance evaluation of all the EWMA charts. The similar results can also be obtained for the other values of $\mathrm{ARL}_{0}$. We have provided the control charts 
Table 4. Run length characteristics of IFNPAS chart for different values of $n$ and $p$ when $\mathrm{ARL}_{0}=370$ and $\lambda=0.05$ and 0.25 .

\begin{tabular}{|c|c|c|c|c|c|c|c|c|c|c|c|c|c|c|c|}
\hline \multirow[b]{2}{*}{$\lambda$} & \multirow[b]{2}{*}{$n$} & & \multicolumn{13}{|c|}{$P$} \\
\hline & & & 0.05 & 0.20 & 0.30 & 0.35 & 0.40 & 0.45 & 0.50 & 0.55 & 0.60 & 0.65 & 0.70 & 0.80 & 0.95 \\
\hline \multirow{12}{*}{0.05} & 5 & ARL & 1.03 & 1.75 & 4.10 & 7.73 & 17.35 & 63.19 & 370.37 & 63.63 & 17.39 & 7.65 & 4.07 & 1.74 & 1.03 \\
\hline & & MDRL & 1.00 & 1.00 & 1.00 & 2.00 & 3.00 & 8.00 & 45.00 & 9.00 & 3.00 & 2.00 & 1.00 & 1.00 & 1.00 \\
\hline & & SDRL & 0.19 & 1.74 & 5.53 & 10.77 & 25.18 & 97.96 & 603.93 & 99.86 & 24.56 & 10.63 & 5.48 & 1.71 & 0.21 \\
\hline & 7 & ARL & 1.00 & 1.31 & 2.71 & 4.89 & 11.32 & 43.76 & 369.29 & 44.38 & 11.42 & 4.91 & 2.62 & 1.31 & 1.00 \\
\hline & & MDRL & 1.00 & 1.00 & 1.00 & 1.00 & 1.00 & 2.00 & 3.00 & 2.00 & 1.00 & 1.00 & 1.00 & 1.00 & 1.00 \\
\hline & & SDRL & 0.06 & 0.98 & 3.62 & 7.23 & 17.55 & 71.86 & 658.63 & 73.39 & 17.20 & 7.17 & 3.48 & 0.99 & 0.07 \\
\hline & 10 & ARL & 1.00 & 1.16 & 1.97 & 3.49 & 7.97 & 31.62 & 369.92 & 31.62 & 7.96 & 3.40 & 1.93 & 1.16 & 1.00 \\
\hline & & MDRL & 1.00 & 1.00 & 1.00 & 1.00 & 2.00 & 3.00 & 38.50 & 4.00 & 2.00 & 1.00 & 1.00 & 1.00 & 1.00 \\
\hline & & SDRL & 0.03 & 0.50 & 2.14 & 4.64 & 11.55 & 47.79 & 606.97 & 47.78 & 11.39 & 4.52 & 2.07 & 0.50 & 0.03 \\
\hline & 15 & ARL & 1.00 & 1.02 & 1.29 & 1.92 & 4.05 & 16.39 & 370.68 & 16.51 & 3.98 & 1.91 & 1.28 & 1.02 & 1.00 \\
\hline & & MDRL & 1.00 & 1.00 & 1.00 & 1.00 & 1.00 & 1.00 & 1.00 & 1.00 & 1.00 & 1.00 & 1.00 & 1.00 & 1.00 \\
\hline & & SDRL & 0.00 & 0.17 & 1.03 & 2.55 & 6.92 & 31.08 & 816.46 & 31.23 & 6.94 & 2.55 & 1.04 & 0.18 & 0.00 \\
\hline \multirow{12}{*}{0.25} & 5 & ARL & 1.03 & 1.85 & 5.42 & 12.80 & 41.08 & 146.54 & 369.14 & 149.03 & 41.67 & 12.87 & 5.38 & 1.85 & 1.03 \\
\hline & & MDRL & 1.00 & 1.00 & 1.00 & 2.00 & 3.00 & 9.00 & 64.00 & 12.00 & 3.00 & 2.00 & 1.00 & 1.00 & 1.00 \\
\hline & & SDRL & 0.2 & 2.06 & 5 & 22.78 & 72.09 & 249.71 & 60 & 25 & 72 & 23.42 & 9.20 & 2.15 & 0.20 \\
\hline & 7 & ARL & 1.00 & 1.33 & 3.44 & 8.28 & 26.68 & 120.44 & 370.19 & 117.05 & 26.94 & 8.23 & 3.35 & 1.33 & 1.00 \\
\hline & & MDRL & 1.00 & 1.00 & 1.00 & 1.00 & 1.00 & 2.00 & 3.00 & 2.00 & 1.00 & 1.00 & 1.00 & 1.00 & 1.00 \\
\hline & & SDRL & 0.06 & 1.11 & 5.98 & 16.32 & 52.69 & 215.66 & 655.82 & 215.89 & 52.10 & 16.36 & 5.87 & 1.11 & 0.05 \\
\hline & 10 & ARL & 1.00 & 1.19 & 2.31 & 4.95 & 16.22 & 91.82 & 370.69 & 92.65 & 16.47 & 4.93 & 2.29 & 1.19 & 1.00 \\
\hline & & MDRL & 1.00 & 1.00 & 1.00 & 1.00 & 2.00 & 10.00 & 85.00 & 11.00 & 2.00 & 1.00 & 1.00 & 1.00 & 1.00 \\
\hline & & SDRL & 0.03 & 0.61 & 2.97 & 8.21 & 29.05 & 152.83 & 592.49 & 154.93 & 29.72 & 8.33 & 2.99 & 0.64 & 0.02 \\
\hline & 15 & ARL & 1.00 & 1.07 & 1.58 & 2.67 & 7.54 & 49.82 & 370.22 & 50.78 & 7.62 & 2.68 & 1.56 & 1.07 & 1.00 \\
\hline & & MDRL & 1.00 & 1.00 & 1.00 & 1.00 & 2.00 & 6.00 & 102.00 & 7.00 & 2.00 & 1.00 & 1.00 & 1.00 & 1.00 \\
\hline & & SDRL & 0.00 & 0.28 & 1.32 & 3.51 & 12.61 & 83.04 & 574.30 & 84.39 & 12.72 & 3.48 & 1.32 & 0.30 & 0.00 \\
\hline
\end{tabular}

Table 5. EQL and RARL measures for different values of $n$ and $\lambda$ when $\mathrm{ARL}_{0}=370$.

\begin{tabular}{|c|c|c|c|c|c|c|c|c|}
\hline \multirow{3}{*}{$\frac{n}{\text { Charts }}$} & \multicolumn{8}{|c|}{$\lambda=0.05$} \\
\hline & \multicolumn{2}{|c|}{5} & \multicolumn{2}{|c|}{7} & \multicolumn{2}{|c|}{10} & \multicolumn{2}{|c|}{15} \\
\hline & EQL & RARL & EQL & RARL & EQL & RARL & EQL & RARL \\
\hline NPAS & 7.2129 & 3.4271 & 6.1441 & 3.5766 & 5.0283 & 3.3312 & 4.2396 & 3.3723 \\
\hline TNPAS & 2.8850 & 1.6519 & 2.7110 & 1.8528 & 2.1396 & 1.6375 & 1.9349 & 1.6878 \\
\hline FNPAS & 2.5031 & 1.3229 & 1.9921 & 1.2514 & 1.8106 & 1.1858 & 1.7059 & 1.2279 \\
\hline \multirow[t]{2}{*}{ IFNPAS } & 1.9791 & 1.0000 & 1.7752 & 1.0000 & 1.7059 & 1.0000 & 1.6369 & 1.0000 \\
\hline & \multicolumn{8}{|c|}{$\lambda=0.25$} \\
\hline NPAS & 5.4310 & 2.6095 & 4.0051 & 2.5009 & 2.9299 & 2.0641 & 2.4926 & 1.9149 \\
\hline TNPAS & 5.3455 & 2.5517 & 3.3081 & 2.2631 & 2.9280 & 2.0693 & 2.0379 & 1.6834 \\
\hline FNPAS & 2.9300 & 1.4817 & 2.1861 & 1.3308 & 1.8995 & 1.1860 & 1.7370 & 1.1306 \\
\hline IFNPAS & 2.1513 & 1.0000 & 1.8836 & 1.0000 & 1.7762 & 1.0000 & 1.6825 & 1.0000 \\
\hline
\end{tabular}

coefficients $L_{t}$ and $L_{f}$ for different choices of $\mathrm{ARL}_{0}$ in Figures 3 and 4 for TNPAS and FNPAS charts, respectively. These figures can be used to obtain the values of $L_{t}$ and $L_{f}$ to approximate control limits at the desired $\mathrm{ARL}_{0}$ values ranging from 50 to 500 for
TNPAS and FNPAS charts, respectively, at $\lambda=0.05$ and 0.25 . For example, the first plot in Figure 3 shows that $L_{t}=3.615$ approximately gives $\mathrm{ARL}_{0}=370$ for TNPAS chart using $n=7$ and $\lambda=0.25$. Similar plots can also be created for NPAS and IFNPAS charts. 

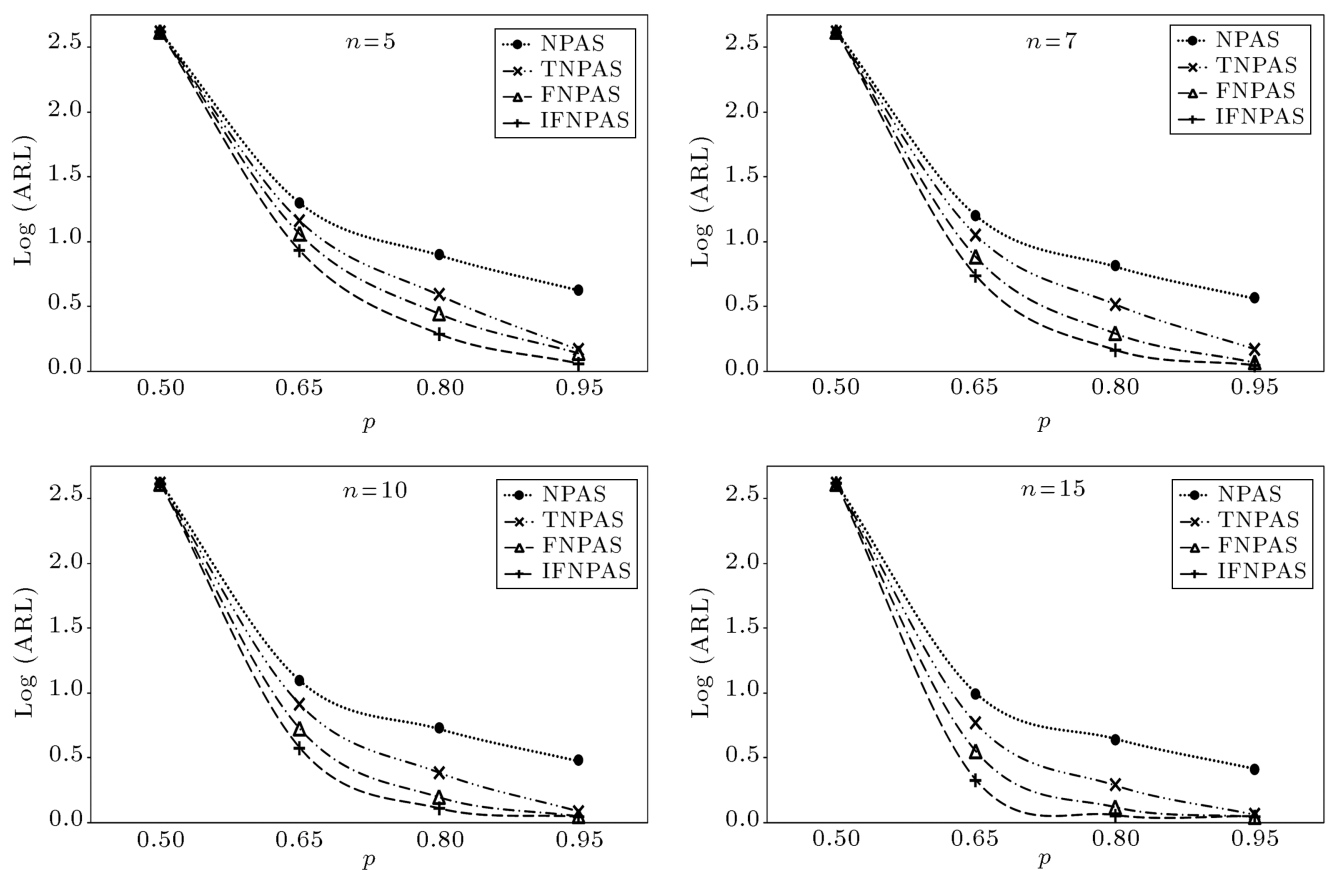

Figure 1. ARL comparison of NPAS, TNPAS, FNPAS, and IFNPAS charts for different values of $n$ when $\lambda=0.05$ and $\mathrm{ARL}_{0}=370$.
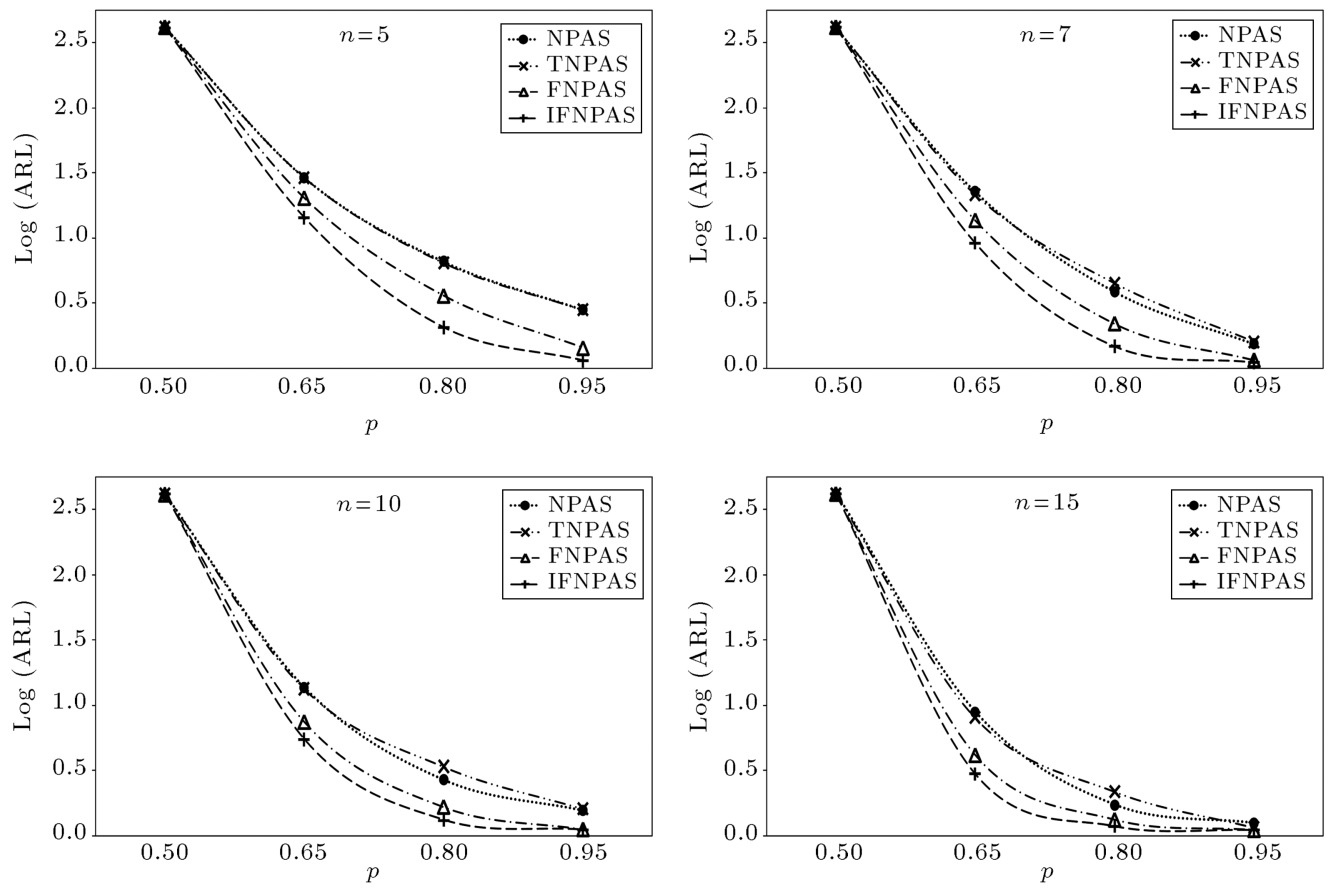

Figure 2. ARL comparison of NPAS, TNPAS, FNPAS, and IFNPAS charts for different values of $n$ when $\lambda=0.25$ and $\mathrm{ARL}_{0}=370$.

Run length characteristics of EWMA charts using FIR without the time varying feature: In order to investigate the effect of FIR feature without the time-varying factor, we have studied the run length characteristics of FNPAS chart without the timevarying factor. The resulting outcomes are presented in Table 6 at $n=7,10$ and $\lambda=0.05,0.25$ for $\mathrm{ARL}_{0}=370$.
These choices are made for a valid comparison with the FNPAS chart using the time-varying run length characteristics provided in Table 3 . By looking at results of Tables 7 and 3, we may observe that timevarying feature improves the performance of FNPAS chart as compared to the chart without time-varying feature at any value of $n$ and $\lambda$. 

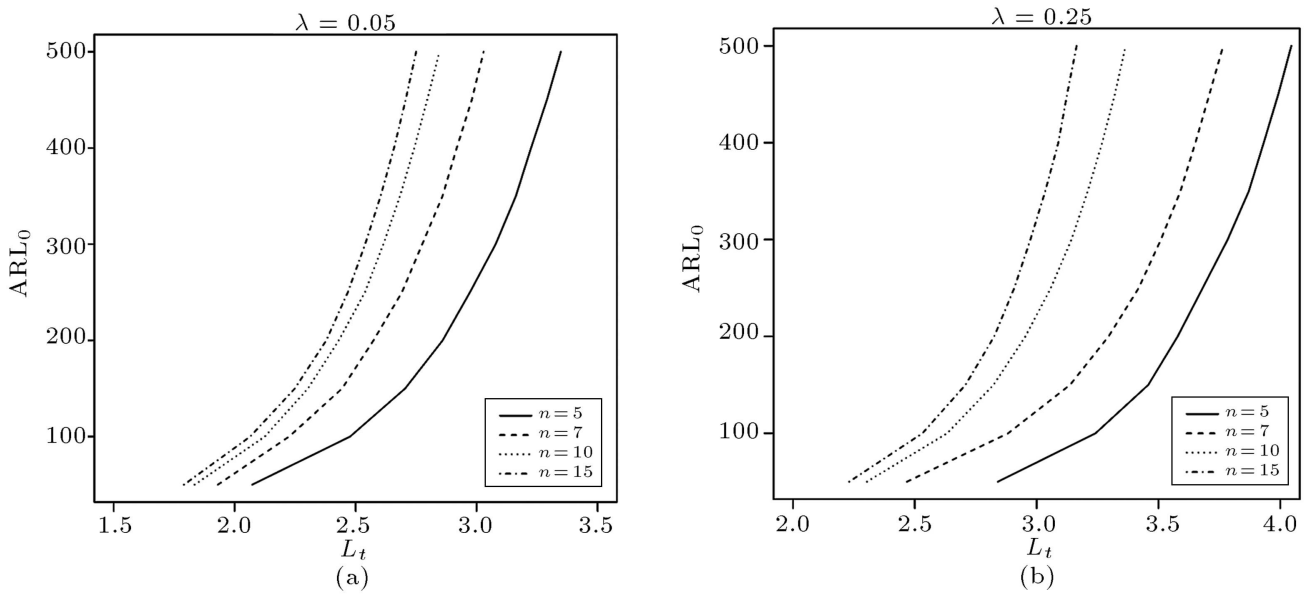

Figure 3. The choice of $L_{t}$ at different $\mathrm{ARL}_{0}$ values for TNPAS chart.
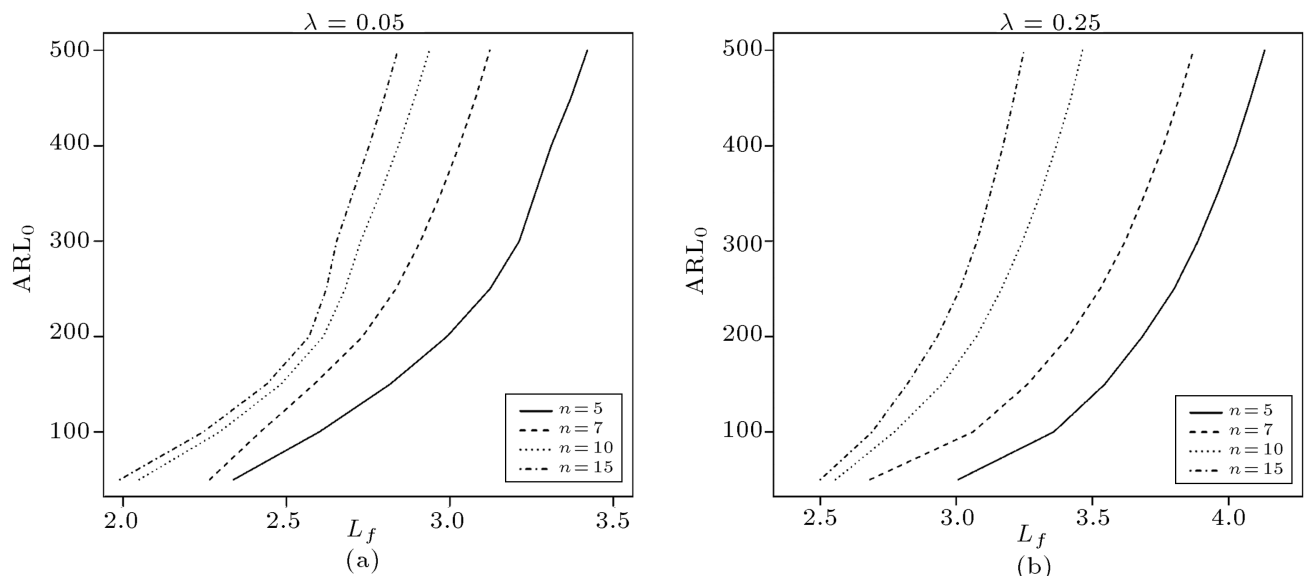

Figure 4. The choice of $L_{f}$ at different $\mathrm{ARL}_{0}$ values for FNPAS chart.

Table 6. Run length characteristics of FNPAS chart without the time-varying features for different values of $n$ and $\lambda$ at $\mathrm{ARL}_{0}=370$.

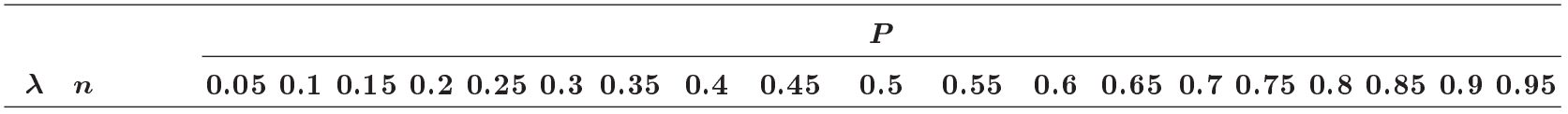

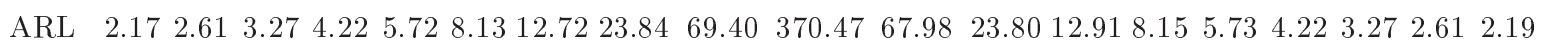

0.057 MDRL 2.00 2.00 3.00 4.00 5.00 8.0012.00 21.00 54.00 261.00 52.00 21.00 12.007 .005 .004 .003 .002 .002 .00

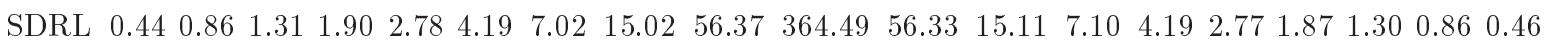

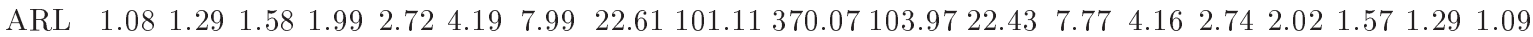

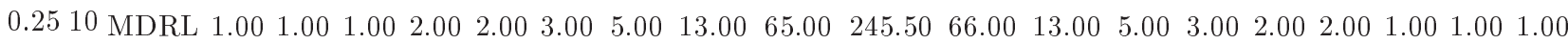

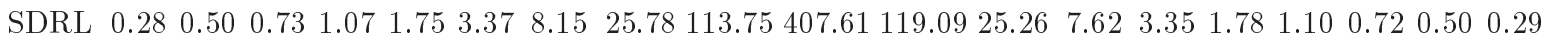

Run length characteristics of EWMA charts (using the FIR feature) at different levels of $f$ : To study the effect of parameter $f$ on the run length performance of EWMA charts using the FIR feature, we have obtained the run length results of FNPAS chart at $n=7$ and $\lambda=0.25$. These are provided in Table 7 . From these results, it can be observed that the performance of FNPAS chart is inversely related to the choice of $f$. It means that FNPAS chart shows better performance at small values of $f$ as compared to the relatively larger values.

Percentile points of the run length distribution: The percentile points of the run length distribution of NPAS, TNPAS, FNPAS, and IFNPAS charts are calculated and provided in Table 8. Based on this table, we can also identify the superior performance of FNPAS and IFNPAS charts as compared to NPAS and 
Table 7. Run length characteristics of FNPAS chart at different levels of $f$ when $n=7, \lambda=0.25$, and $\mathrm{ARL}_{0}=370$.

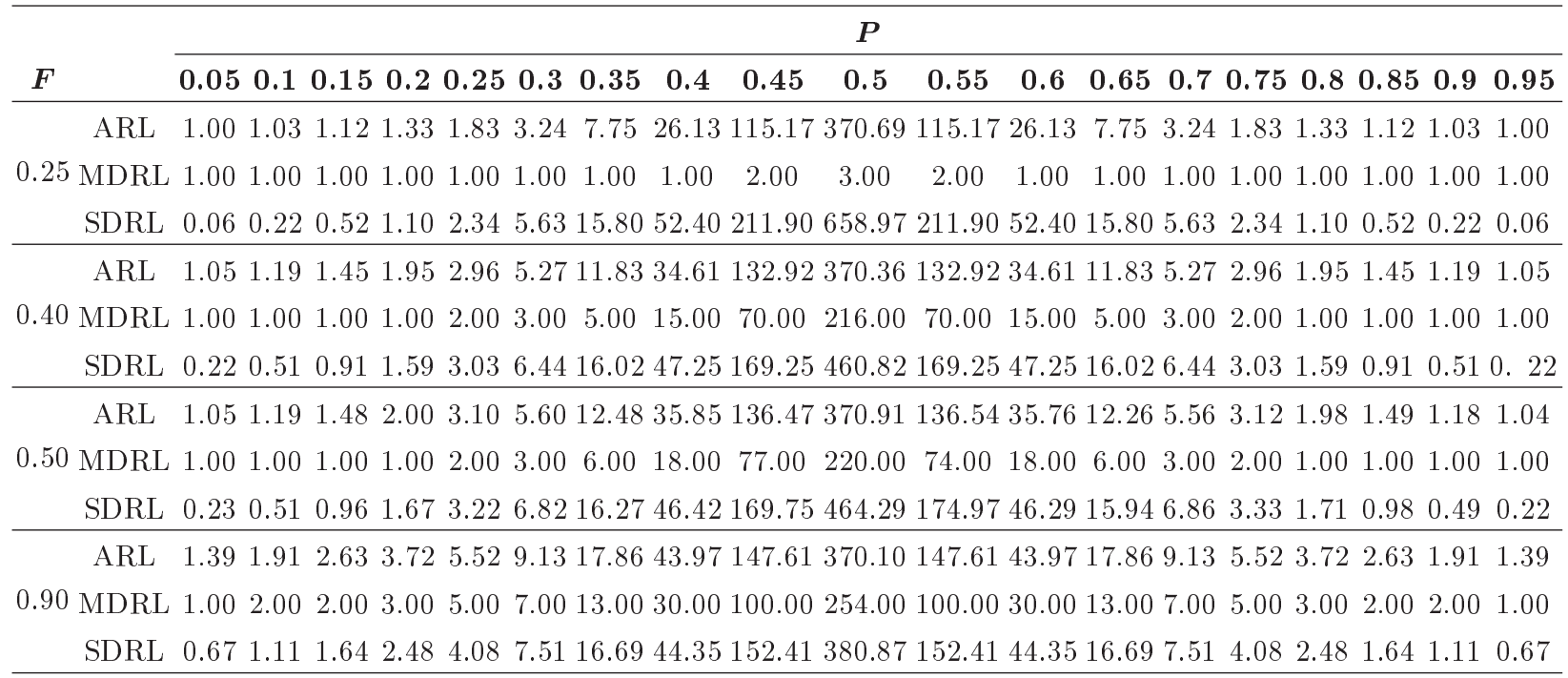

Table 8. Percentiles of the run length distribution for NPAS, TNPAS, FNPAS, and IFNPAS charts when $n=7, \lambda=0.25$, and $f=0.5$.

\begin{tabular}{|c|c|c|c|c|c|c|c|c|c|c|}
\hline \multirow{2}{*}{ Charts } & \multicolumn{10}{|c|}{$p$} \\
\hline & Percentile point & 0.30 & 0.35 & 0.40 & 0.45 & 0.50 & 0.55 & 0.60 & 0.65 & 0.70 \\
\hline \multirow{7}{*}{ NPAS } & 1 & 2 & 2 & 3 & 4 & 6 & 4 & 3 & 2 & 2 \\
\hline & 5 & 3 & 4 & 6 & 11 & 21 & 11 & 6 & 4 & 3 \\
\hline & 25 & 6 & 9 & 17 & 45 & 108 & 45 & 17 & 9 & 6 \\
\hline & 50 & 9 & 16 & 34 & 105 & 257 & 105 & 34 & 16 & 9 \\
\hline & 75 & 14 & 27 & 65 & 207.25 & 505 & 207.25 & 65 & 27 & 14 \\
\hline & 95 & 26 & 54 & 134 & 445.05 & 1091 & 445.05 & 134 & 54 & 26 \\
\hline & 99 & 38 & 79 & 208 & 681.01 & 1730.03 & 681.01 & 208 & 79 & 38 \\
\hline \multirow{7}{*}{ TNPAS } & 1 & 1 & 1 & 1 & 1 & 1 & 1 & 1 & 1 & 1 \\
\hline & 5 & 1 & 1 & 3 & 6 & 13 & 6 & 3 & 1 & 1 \\
\hline & 25 & 4 & 7 & 15 & 41 & 102 & 41 & 15 & 7 & 4 \\
\hline & 50 & 8 & 14 & 32 & 102 & 254 & 102 & 32 & 14 & 8 \\
\hline & 75 & 13 & 26 & 63 & 206 & 507.25 & 206 & 63 & 26 & 13 \\
\hline & 95 & 25 & 53 & 133 & 447.05 & 1104.05 & 447.05 & 133 & 53 & 25 \\
\hline & 99 & 37 & 78 & 206.01 & 692.01 & 1768.01 & 692.01 & 206.01 & 78 & 37 \\
\hline \multirow{7}{*}{ FNPAS } & 1 & 1 & 1 & 1 & 1 & 1 & 1 & 1 & 1 & 1 \\
\hline & 5 & 1 & 1 & 1 & 1 & 1 & 1 & 1 & 1 & 1 \\
\hline & 25 & 1 & 1 & 3 & 8 & 27.75 & 8 & 3 & 1 & 1 \\
\hline & 50 & 3 & 6 & 17 & 73 & 218 & 73 & 17 & 6 & 3 \\
\hline & 75 & 7 & 17 & 51 & 196 & 537.25 & 196 & 51 & 17 & 7 \\
\hline & 95 & 20 & 47 & 130.05 & 482.05 & 1297.15 & 482.05 & 130.05 & 47 & 20 \\
\hline & 99 & 31 & 75 & 214.01 & 760 & 1998.03 & 760 & 214.01 & 75 & 31 \\
\hline \multirow{7}{*}{ IFNPAS } & 1 & 1 & 1 & 1 & 1 & 1 & 1 & 1 & 1 & 1 \\
\hline & 5 & 1 & 1 & 1 & 1 & 1 & 1 & 1 & 1 & 1 \\
\hline & 25 & 1 & 1 & 1 & 1 & 1 & 1 & 1 & 1 & 1 \\
\hline & 50 & 1 & 1 & 1 & 2 & 3 & 2 & 1 & 1 & 1 \\
\hline & 75 & 2 & 6 & 27 & 146 & 497.25 & 146 & 27 & 6 & 2 \\
\hline & 95 & 14 & 40 & 134 & 570.05 & 1731 & 570.05 & 134 & 40 & 14 \\
\hline & 99 & 28 & 76 & 245.01 & 955 & 3002.18 & 955 & 245.01 & 76 & 28 \\
\hline
\end{tabular}

TNPAS charts. For example, the 25th percentile point of IFNPAS chart is 1 at $p=0.30$, whereas the same percentile for NPAS chart is 6 . It clearly shows the added advantage of using IFNPAS chart as compared to NPAS chart. Similar comparisons can also be made for the other percentile points.

\section{Illustrative example}

In order to illustrate the application of under-study charts, the same example is used here as was used earlier by [42] and [18] from [19]. The filling volume of soft-drink beverage bottles is an important quality 
Table 9. EWMA statistic and the control limits for different charts.

\begin{tabular}{|c|c|c|c|c|c|c|c|c|c|}
\hline \multirow[b]{3}{*}{ Sample } & \multirow[b]{3}{*}{ EWMA } & \multicolumn{8}{|c|}{ Control limits } \\
\hline & & \multicolumn{2}{|c|}{ NPAS Chart } & \multirow{2}{*}{$\begin{array}{l}\text { TNPAS } \\
\text { LCL }\end{array}$} & \multirow{2}{*}{$\begin{array}{l}\text { Chart } \\
\text { UCL }\end{array}$} & \multirow{2}{*}{$\begin{array}{l}\text { FNPAS } \\
\text { LCL }\end{array}$} & \multirow{2}{*}{$\begin{array}{c}\text { Chart } \\
\text { UCL }\end{array}$} & \multirow{2}{*}{$\begin{array}{l}\text { IFNPAS } \\
\text { LCL }\end{array}$} & \multirow{2}{*}{$\begin{array}{l}\text { Chart } \\
\text { UCL }\end{array}$} \\
\hline & & LCL & UCL & & & & & & \\
\hline 1 & 0.7957 & 0.7224 & 0.8484 & 0.7640 & 0.8068 & 0.7743 & 0.7965 & 0.7795 & 0.7913 \\
\hline 2 & 0.8002 & 0.7224 & 0.8484 & 0.7559 & 0.8149 & 0.7672 & 0.8036 & 0.7706 & 0.8002 \\
\hline 3 & 0.7944 & 0.7224 & 0.8484 & 0.7501 & 0.8207 & 0.7609 & 0.8099 & 0.7627 & 0.8081 \\
\hline 4 & 0.7779 & 0.7224 & 0.8484 & 0.7456 & 0.8252 & 0.7552 & 0.8156 & 0.7559 & 0.8149 \\
\hline 5 & 0.7622 & 0.7224 & 0.8484 & 0.7420 & 0.8288 & 0.7501 & 0.8207 & 0.7499 & 0.8209 \\
\hline 6 & 0.7583 & 0.7224 & 0.8484 & 0.7389 & 0.8319 & 0.7457 & 0.8251 & 0.7448 & 0.8260 \\
\hline 7 & 0.7494 & 0.7224 & 0.8484 & 0.7363 & 0.8345 & 0.7418 & 0.8290 & 0.7403 & 0.8305 \\
\hline 8 & 0.7351 & 0.7224 & 0.8484 & 0.7341 & 0.8367 & 0.7384 & 0.8324 & 0.7365 & 0.8343 \\
\hline 9 & 0.7376 & 0.7224 & 0.8484 & 0.7321 & 0.8386 & 0.7354 & 0.8354 & 0.7331 & 0.8377 \\
\hline 10 & 0.7297 & 0.7224 & 0.8484 & 0.7305 & 0.8403 & 0.7328 & 0.8380 & 0.7302 & 0.8405 \\
\hline 11 & 0.7275 & 0.7224 & 0.8484 & 0.7290 & 0.8418 & 0.7305 & 0.8403 & 0.7278 & 0.8430 \\
\hline 12 & 0.7201 & 0.7224 & 0.8484 & 0.7277 & 0.8431 & 0.7286 & 0.8422 & 0.7256 & 0.8452 \\
\hline 13 & 0.7072 & 0.7224 & 0.8484 & 0.7265 & 0.8443 & 0.7268 & 0.8440 & 0.7237 & 0.8471 \\
\hline 14 & 0.7061 & 0.7224 & 0.8484 & 0.7255 & 0.8453 & 0.7253 & 0.8455 & 0.7221 & 0.8487 \\
\hline 15 & 0.7101 & 0.7224 & 0.8484 & 0.7246 & 0.8462 & 0.7240 & 0.8468 & 0.7207 & 0.8501 \\
\hline
\end{tabular}

characteristic that is measured (approximately) by placing a gauge over the crown. The control limits of NPAS, TNPAS, FNPAS, and IFNPAS charts compare the height of the liquid in the neck of the bottle against a coded scale at which a reading of zero corresponds to the correct filling height. Fifteen samples of size $n=10$ with $\lambda=0.25$ have been analyzed and the data set with the EWMA statistic $W_{t}$ and variable $M$ are plotted in Figure 5. In this figure, $X_{1}, X_{2}, \ldots, X_{10}$ represent the observations in each sample. The control chart multipliers, $L_{a}=2.490, L_{t}=2.709, L_{f}=2.811$, and $L_{i f}=3.486$, have been used for the NPAS, TNPAS, FNPAS, and IFNPAS charts, respectively, that give the desired $\mathrm{ARL}_{0}=370$ for each chart. The EWMA statistic with control limits for all charts has been reported in Table 9 and plotted in Figure 6.

It can be observed from Figure 6 that the control limits of the TNPAS chart converge with the limits of NPAS chart as the number of samples increases, while the EWMA control limits of the FNPAS and IFNPAS charts are always narrower than the other two charts. We can clearly see that the FNPAS and IFNPAS charts have better detection abilities as compared to the other two charts as the out-of-control signal is detected at the 8th sample by the FNPAS and IFNPAS charts, at the 10th sample by the TNPAS chart and at the 12 th sample by the NPAS chart. This simple example clearly shows the benefit of using the time-varying and FIR-based limits for the NPEWMA control charts as compared to the asymptotic limits.

\section{Conclusions and recommendations}

In this study, we investigated the performance of NPEWMA chart using asymptotic, time-varying, and FIR-based control limits. Computations have been performed using NPAS chart proposed by Yang et al. [18]; these results can be generalized for the other nonparametric charts. The comparisons among under-study charts have been made on the basis of run length characteristics with some effective performance measures. It has been observed that the ability of the NPEWMA chart to detect shifts in process target can be improved by using exact (time-varying limits) instead of asymptotic control limits, particularly for smaller values of smoothing parameter $\lambda$. The FIR feature has been also shown to contribute significantly to further increase the sensitivity of NPEWMA chart to detect shifts in 


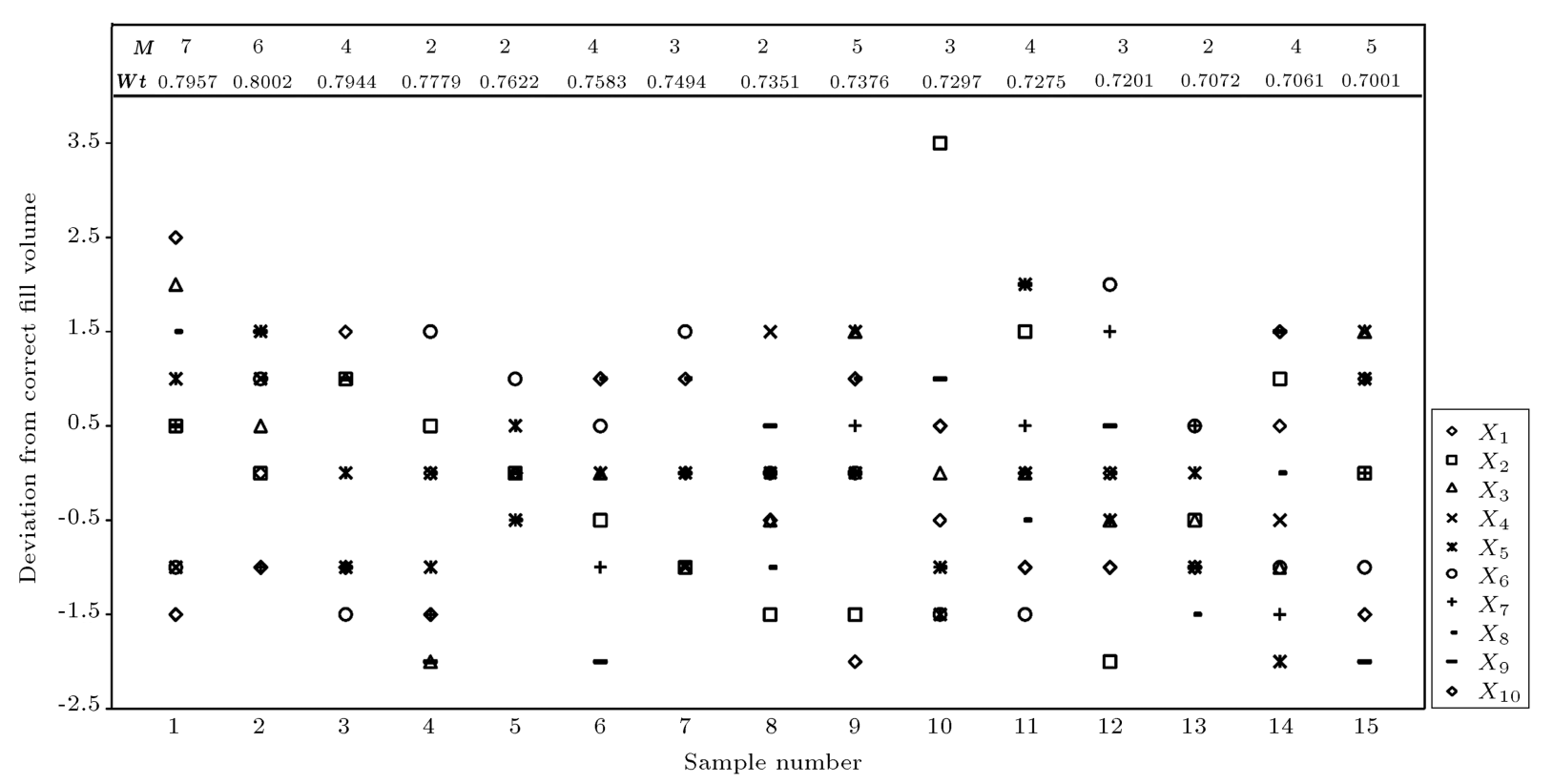

Figure 5. Plot of data values with $M$ and $W_{t}$ statistics.

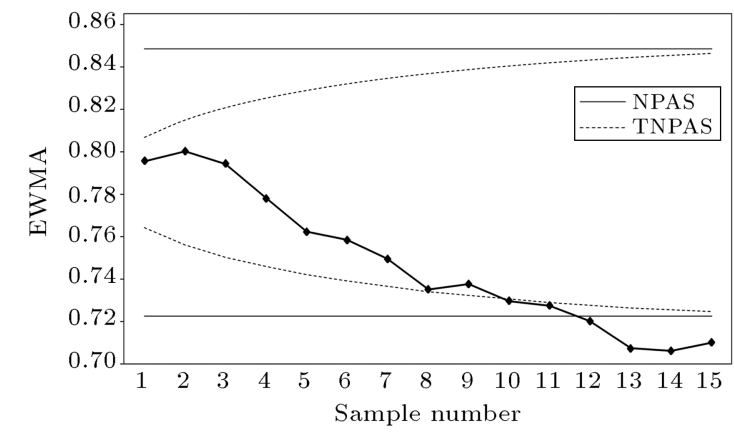

(a)

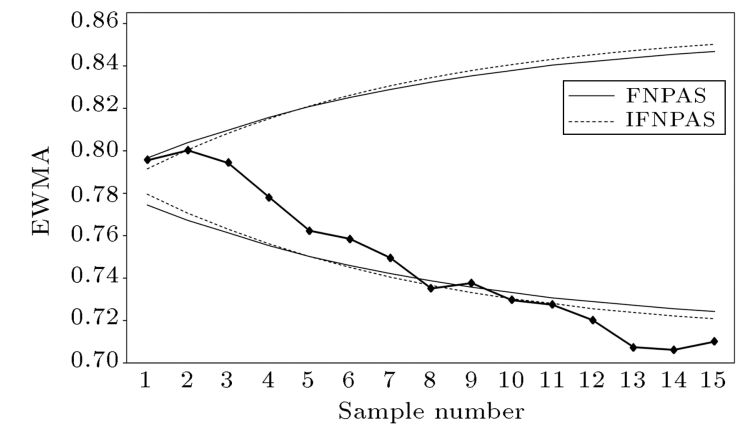

(b)

Figure 6. Control charts display for NPAS, TNPAS, FNPAS, and IFNPAS charts.

process target. In future, this study can be helpful for quality practitioners to choose a more sensitive NPEWMA chart for monitoring process target.

The scope of this study can be extended to the design structures of other control charts such as mixed EWMA CUSUM [43], combined ShewhartEWMA [44], Shewhart-CUSUM [45], and double EWMA charts [46]. Moreover, these ideas may also be investigated for multivariate setups [47].

\section{Acknowledgments}

The authors are thankful to the anonymous reviewers for their valuable comments that help to improve the initial version of the article. The authors Saddam Akber Abbasi and Muhammad Riaz would like to acknowledge the support provided by the Deanship of Scientific Research at King Fahd University of Petroleum \& Minerals (KFUPM) under Research Grant IN131019.

\section{References}

1. Shewhart, W.A., Economic Control of Quality Manufactured Product. D. Van Nostrand, New York, reprinted by the American Society for Quality Control in 1980, Milwauker, WI (1931).

2. Page, E.S. "Continuous inspection schemes", Biometrika, 41(1-2), pp. 100-115 (1954).

3. Roberts, S.W. "Control chart tests based on geometric moving averages", Technometrics, 1(3), pp. 239-250 (1959).

4. Amin, R.W., Reynolds Junior, M.R. and Baker, S.T. "Nonparametric quality control charts based on the sign statistic", Communications in Statistics-Theory and Methods, 24, pp. 1597-1624 (1995).

5. Chakraborti, S., Van der Laan, P. and Bakir, S.T. "Nonparametric control charts: an overview and some results", J. Qual. Tech., 33(3), pp. 304-315 (2001).

6. Altukife, F.S. "A new nonparametric control charts based on the observations exceeding the grand me- 
dian", Pakistan Journal of Statistics, 19(3), pp. 343351 (2003).

7. Das, N. "A comparison study of three non-parametric control charts to detect shift in location parameters", Int. J. Adv. Manuf. Technol., 41(7-8), pp. 799-807 (2009).

8. Qiu, P., Zou, C. and Wang, Z. "Nonparametric profile monitoring by mixed effects modeling", Technometrics, 52(3), pp. 265-277 (2010). 10.1198/TECH.2010.08188

9. Human, S.W., Chakraborti, S. and Smit, C.F. "Nonparametric shewhart-type sign control charts based on runs", Communications in Statistics -Theory and Methods, 39(11), pp. 2046-2062 (2010).

10. Khilare, S.K. and Shirke, D.T. "A nonparametric synthetic control chart using sign statistic", Communications in Statistics - Theory and Methods, 39(18), pp. 3282-3293 (2010).

11. Pawar, V.Y. and Shirke, D.T. "A nonparametric shewhart-type synthetic control chart", Communications in Statistics-Simulation and Computation, 39(8), pp. 1493-1505 (2010).

12. Yang, S. and Cheng, S. "A new non-parametric CUSUM mean chart", Quality and Reliability Engineering International, 27(7), pp. 867-875 (2011).

13. Yang, S.F., Cheng, T.C., Huang, Y.C. and Cheng, S. "A new chart for monitoring service process mean", Quality and Reliability Engineering International, 28(4), pp. 377-386 (2012).

14. Yan, S.F. "An improved distribution-free EWMA mean chart", Communications in Statistics-Simulation and Computation, 44, pp. 1-18 (2015).

15. Li, S.Y., Tang, L.C. and Ng, S.H. "Nonparametric CUSUM and EWMA control charts for detecting mean shifts", J Qual. Tech., 42(2), pp. 209-226 (2010).

16. Zou, C. and Tsung, F. "A multivariate signewma control chart", Technometrics, 53(1), pp. 84-97 (2011).

17. Graham, M.A., Chakraborti, S. and Human, S.W. "A nonparametric EWMA sign chartforlocation based on individual measurements", Quality Engineering, 23(3), pp. 227-241 (2011).

18. Yang, S.F., Lin, J.S. and Cheng, S.W. "A new nonparametric ewma sign control chart", Expert Systems with Applications, 38(5), pp. 6239-6243 (2011).

19. Montgomery, D.C., Introduction to Statistical Quality Control, 6th Ed., New York, John Willey \& Sons (2009).

20. Steiner, S.H. "EWMA control charts with time-varying control limits and fast initial response", J. Qual. Tech., 31(1), pp. 75-86 (1999).

21. Haq, A., Brown, J. and Moltchanova, E. "Improved fast initial response features for exponentially weighted moving average and cumulative sum control charts", Quality and Reliability Engineering International, 30(5), pp. 697-710 (2014).
22. Lucas, J.M. and Crosier, R.B. "Fast initial response for CUSUM quality control schemes: Give your CUSUM a head start", Technometrics, 24(3), pp. 199-205 (1982).

23. Lucas, J.M. and Saccucci, M.S. "Exponentially weighted moving average control schemes: Properties and enhancements", Technometrics, 32(1), pp. 1-12 (1990).

24. Rhoads, T.R., Montgomery, D.C. and Mastrangelo, C.M. "A fast initial response scheme for the exponentially weighted moving average control chart", Quality Engineering, 9(2), pp. 317-327 (1996). $10.1080 / 08982119608919048$

25. Chiu, W.C. "Generally weighted moving average control charts with fast initial response features", Journal of Applied Statistics, 36(3), pp. 255-275 (2009).

26. Zhang, S. and Wu, Z. "Monitoring the process mean and variance by the WLC scheme with variable sampling intervals", IIE Trans., 38(4), pp. 377-387 (2006).

27. Wu, Z., Jiao, J.X., Yang, M., Liu, Y. and Wang, Z.J. "An enhanced adaptive CUSUM control chart", IIE Trans., 41(7), pp. 642-653 (2009).

28. Ryu, J.H., Wan, H. and Kim, S. "Optimal design of a CUSUM chart for a mean shift of unknown size", $J$. Qual. Tech., 42(3), pp. 311-326 (2010).

29. Ou, Y.J., Wu, Z. and Goh, T.N. "A new sprt chart for monitoring process mean and variance", Int. J. of Production Economics, 132(2), pp. 303-314 (2011).

30. Ou, Y.J., Wu, Z. and Tsung, F. "A comparison study of effectiveness and robustness of control charts for monitoring process mean", Int. J. of Production Economics, 135(1), pp. 479-490 (2012).

31. Ahmad, S., Riaz, M., Abbasi, S.A. and Lin, Z. "On median control charting under double sampling scheme", European Journal of Industrial Engineering, 8(4), pp. 478-512 (2014).

32. Ahmad, S., Riaz, M., Abbasi, S.A. and Lin, Z. "On monitoring process variability under double sampling scheme", Int. J. of Production Economics, 142(2), pp. 388-400 (2013). DOI: 10.1016/j.ijpe.2012.12.015

33. Ahmad, S. and Riaz, M. "Process monitoring using quantiles control charts", Journal of Testing and Evaluation, 42(4), pp. 962-979 (2014).

34. Ahmad, S., Abbasi, S.A., Riaz, M. and Abbas, N. "On efficient use of auxiliary information for control charting in SPC", Comput. Ind. Eng., 67, pp. 173-184 (2014). 10.1016/j.cie.2013.11.004

35. Ahmad, S., Riaz, M., Abbasi, S.A. and Lin, Z. "On efficient median control charting", Journal of the Chinese Institute of Engineers, 37(3), pp. 358-375 (2014).

36. Bashiri, M., Amiri, A., Doroudyan, M.H. and Asgari, A. "Multi-objective genetic algorithm for economic statistical design of Xbar control chart", Scientia Iranica, 20(3), pp. 909-918 (2013). 
37. Maravelakis, P., Panaretos, J. and Psarakis, S. "An examination of the robustness to non normality of the EWMA control charts for the dispersion", Communications in Statistics: Simulation and Computation, 34(4), pp. 1069-1079 (2005).

38. Abbasi, S.A. "On the performance of EWMA charts in presence of two component measurement error", Quality Engineering, 22(3), pp. 199-213 (2010).

39. Abbasi, S.A. and Miller, A. "MDEWMA chart: An efficient and robust alternative to monitor process dispersion", Journal of Statistical Computation and Simulation, 83(2), pp. 247-268 (2013).

40. Kim, M.J., Number of Replications Required in Control Chart Monte Carlo Simulation Studies, University of Northern Colorado, USA (2005).

41. Schaffer, J.R. and Kim, M.J. "Number of replications required in control chart Monte Carlo simulation studies", Commun. Stat. Simulation C., 36(5), pp. 10751087 (2007).

42. Abbasi, S.A., Miller, A. and Riaz, M. "Nonparametric progressive control chart for monitoring process target", Quality and Reliability Engineering International, 29(7), pp. 1069-1080 (2013).

43. Abbas, N., Riaz, M. and Does, R.J.M. "Mixed exponentially weighted moving average-cumulative sum charts for process monitoring", Quality and Reliability Engineering International, 29(3), pp. 345-356 (2013).

44. Abujiya, M.R., Riaz, M. and Lee, M.H. "Enhancing the performance of combined shewhart-EWMA charts", Quality and Reliability Engineering International, 29(8), pp. 1093-1106 (2013).

45. Lucas, J.M. "Combined shewhart-CUSUM quality control schemes", J. Qual. Tech., 14(2), pp. 51-59 (1982).

46. Zhang, L. and Chen, G. "An extended EWMA mean Chart", Quality Technology and Quantitative Management, 2(1), pp. 39-52 (2005).

47. Khoo, B.C. "Increasing the sensitivity of multivariate EWMA control chart", Quality Engineering, 16(1), pp. $75-85$ (2003).

\section{Biographies}

Saddam Akber Abbasi obtained his BS (Honors) degree in Computer Science from the PMAS-Arid Agriculture University Rawalpindi, Pakistan in 2005 and his MSc degree in Statistics from Quaid-i-Azam
University, Islamabad, Pakistan in 2007. He obtained his $\mathrm{PhD}$ degree in Statistics at the Department of Statistics, the University of Auckland, Auckland, New Zealand in 2012.

$\mathrm{He}$ is serving as an Assistant Professor in the Department of Mathematics, Statistics and Physics, Qatar University, Doha, Qatar. Before joining Qatar University, he served King fahd University of Petroleum and Minerals, Dhahran, KSA for 3 years.

His research interests include Statistical Process Control (SPC) and artificial neural networks.

Shabbir Ahmad obtained his MSc degree in Statistics from the PMAS-Arid Agriculture University Rawalpindi, Pakistan in 2002 and his MPhil degree in Statistics from Quaid-i-Azam University, Islamabad, Pakistan in 2005. He served as a Statistical Officer in National Accounts Wing, Pakistan Bureau of Statistics (PBS) during 2006-2007. He obtained his $\mathrm{PhD}$ degree in Statistics at the Department of Mathematics, Institute of Statistics, Zhejiang University, Hangzhou, China in 2013. He is serving as an Assistant Professor in the Department of Mathematics, COMSATS Institute of Information Technology, Wah Cantt, Pakistan. His current research interests include Statistical Process Control (SPC) and Application of Sampling Techniques.

Muhammad Riaz earned the MSc degree in Statistics from the Department of Mathematics and Statistics Quaid-i-Azam University Islamabad Pakistan in 2001 and the PhD in Statistics from the Institute of Business and Industrial Statistics University of Amsterdam, The Netherlands, in 2008. He served as a Statistical Officer in MINFAL Pakistan during 2002-2003, as a Staff Demographer in PIDE Pakistan during 2003-2004, as a Lecturer in the Department of Statistics, Quaid-i-Azam University, Islamabad, Pakistan, during 2004-2007, as an Assistant Professor in the Department of Statistics, Quaid-i-Azam University, Islamabad, Pakistan during 2007-2010. He is serving as an Assistant Professor in the Department of Mathematics and Statistics, King Fahad University of Petroleum and Minerals, Dhahran 31261, Saudi Arabia, from 2010-Present. His current research interests include statistical process control, non-parametric techniques, experimental designs, and computational statistics (focusing code development). 